\title{
Improved Trust Establishment Performance with Transaction-based Preprocessing
}

\author{
Julian Templeton ${ }^{\dagger, *}$, Thomas $\operatorname{Tran}^{\dagger}$ \\ ${ }^{\dagger}$ Electrical Engineering and Computer Science, University of Ottawa, Canada
}

\begin{abstract}
In Multi-Agent Systems, trustees can utilize trust establishment models to help improve their trust with trustors in the environment. This improved trust helps to improve the quality of interactions in the environment and increases the viability of a trustee as an interaction partner. To help current and future trust establishment models become more modular to adapt to changes brought by future research and to introduce a method to allow for improved performance, a generalized trust establishment model architecture is presented. The proposed Generalized Trust Establishment Model is a simplistic model which illustrates how the generalized architecture can be used to design a competent trust establishment model. This model uses the architecture's newly proposed transaction-level preprocessing module to achieve strong simulation results. This preprocessing module allows a model to fine-tune the amount of resources that will be given to trustors before the transactions occur to help more accurately meet the needs of trustors. Using the preprocessing module, the proposed model better meets a trustor's needs and achieves a higher average trust in the environment faster than when the preprocessing is not performed. This exhibits that preprocessing is an important technique that can be used by any trust establishment model to improve the model's performance.

Keywords: Trust establishment, Multi-Agent Systems, Intelligent agents
\end{abstract}

\section{Introduction}

Autonomous agents are becoming increasingly intelligent in Multi-Agent Systems (MASs) and hence the techniques used by these agents are growing in complexity. Due to this, these agents are referred to as intelligent agents and utilize a variety of tools from a trust management module to help with their decision-making abilities when collaborating with other agents in MASs [1]. The trust management module from [2] proposes that agents should be able to both evaluate the trust of another agent with a trust evaluation model and establish their own trustworthiness in the environment with a trust establishment model. Currently, several trust establishment models have been proposed which help agents increase their trust in MASs without using too many resources, referred to as Utility Gain (UG), for the improved trust. The service providing agents which desire to establish trust are referred to as trustees and the agents who consume services are referred to as trustors.

As trust establishment model complexity rises, it becomes more challenging to implement and optimize the models. Depending on a model's design, it may also be challenging for the model to adapt to new research ideas. Thus, this paper presents a generalized trust establishment model architecture which encapsulates the design possibilities from existing models and future research to allow existing and future models to be designed with the same structure. This architecture introduces a new transaction-level preprocessing module which can increase a model's performance by fine-tuning the UG proposed or provided to trustors before each transaction, rather than performing updates after transactions using trustor feedback. A new trust establishment model is designed with the architecture and includes a togglable preprocessing module. This model will be compared to existing models in simulated tests to highlight the advantages gained by using the new preprocessing module.

*jtemp005@uottawa.ca

This article is () 2021 by author(s) as listed above. The article is licensed under a Creative Commons Attribution (CC BY 4.0) International license (https://creativecommons.org/licenses/by/4.0/legalcode), except where otherwise indicated with respect to particular material included in the article. The article should be attributed to the author(s) identified above. 


\section{Background Information}

We will first describe the Integrated Trust Establishment (ITE) model [3] to exhibit how a model can be structured and to display specific ideas that will be used later in this paper. ITE uses trustor retention, Q-Learning, indirect and direct feedback, and dynamic hyperparameters to achieve high trust values while minimizing the UG being provided. Many of ITE's ideas originate from the Reinforcement Learning Based Trust Establishment (RLTE) model [4] and the Acting as a Trustee Using Implicit Feedback (ATeIF) model [5].

To predict how a trustor weighs each of the criteria $c_{i}$ of a task s, ITE stores predicted relative weight $(\mathrm{rw})$ values between trustee y and trustor $\mathrm{x}$, represented as $r w_{x}^{y}\left(s_{c_{i}}\right)$. Trustors contain the actual rw values for $s_{c_{i}}$, which equal to a criterion's weight $w_{x}\left(s_{c_{i}}\right)$ divided by the demand for that criterion $d_{x}\left(s_{c_{i}}\right)$. With these and other values, ITE calculates the UG to explicitly and implicitly provide to $\mathrm{x}$ for $s_{c_{i}}$. The weighted combination of these explicit and implicit values represents the total improvement to provide for a criterion, denoted by Improvement $t_{x}^{y}\left(s_{c_{i}}\right)$. Using this, the UG values to provide for each criterion are calculated.

$$
u g_{x}^{y}\left(s_{c_{i}}, \text { req }\right)=\text { Improvement }_{x}^{y}\left(s_{c_{i}}, r e q\right)+\operatorname{MinUG}^{y}\left(s_{c_{i}}\right)
$$

- Where $u g_{x}^{y}\left(s_{c_{i}}, r e q\right)$ is the UG that is proposed to $\mathrm{x}$ by y for $s_{c_{i}}$ during the interaction req and where $\operatorname{MinU} G^{y}\left(s_{c_{i}}\right)$ is the minimum UG value which y can provide for $s_{c_{i}}$

The trustee then provides the trustor with each $u g_{x}^{y}\left(s_{c_{i}}, r e q\right)$ value for the $\mathrm{p}$ criteria of task s alongside the total $\mathrm{UG}$, denoted by $U G_{x}^{y}(s, r e q)$, which is the summation of all $u g_{x}^{y}\left(s_{c_{i}}, r e q\right)$ values. After the UG is provided, the trustor may send their satisfaction with the transaction to the trustee using equation (2.2). This feedback can also be predicted if not provided. Finally, ITE will use this direct feedback to adjust the trustee's behaviour to balance the amount of provided UG with a high enough trust value.

$$
S A T_{x}^{y}(s, \text { tra })=\sum_{i=1}^{p} \frac{w_{x}\left(s_{c_{i}}\right) * u g_{x}^{y}\left(s_{c_{i}}, r e q\right)}{d_{x}\left(s_{c_{i}}\right)}
$$

- Where $S A T_{x}^{y}(s, \operatorname{tra})$ is trustor x's satisfaction with transaction tra

\section{Generalized Trust Establishment Model Architecture}

To provide context on what the architecture must encapsulate, we briefly outline some of the techniques and designs used by existing models. The Fuzzy Logic Based Trust Establishment Model for Intelligent Agents (FTE) [6], like ITE, is based on RLTE's design, but uses fuzzy logic systems to learn. An earlier model presented in [7] analyzes the potential impact that a successful or unsuccessful transaction can have on a trustee's reputation to then adjust the trustee's behaviour. Another early model, which we refer to as ETME, assigns classification types to trustors, reclassifying them after unsuccessful transactions, and provides them a UG based on their classification [8]. Unlike ITE, ATeIF, FTE, and RLTE which all handle multiple criteria tasks, both earlier models only handle single criteria tasks.

From the above we see that current models use the data of completed transactions and the corresponding feedback to learn how to improve a trustee's trust. Two future research topics which must be considered are the social relationships of agents, which may help perform more robust calculations, and trustor attack detection, which can be an internal or external module that helps a trustee avoid being abused by malicious trustors for improved trust.

Since current trust establishment models alter a trustee's behaviour with data obtained after transactions occur and use feedback to make the updates, the learning process can be defined as the act of trustor-level postprocessing. Existing models can be designed under this trustor-level postprocessing module. When examining existing research to find finetuning approaches which can support this postprocessing, the SenseBERT model's use of 
fine-tuning to achieve improved performance at a word sense disambiguation task seems appropriate for trust establishment [9]. This idea allows a model to use a preprocessing module, in addition to a postprocessing module, that can improve the model's performance.

Thus, the generalized trust establishment model architecture contains both transactionlevel preprocessing and trustor-level postprocessing modules. The transaction-level preprocessing module presented here learns to predict some output and uses that prediction to fine-tune the UG values to be proposed or provided to a trustor. Online Machine Learning algorithms can help a model use transaction-based input data to learn something regarding a task that will be used to adjust the UG values. These algorithms learn patterns regarding behaviours surrounding specific tasks, rather than of trustors, by using infinite data streams. This results in transaction-level preprocessing and will be utilized in the next section.

\section{Generalized Trust Establishment Model}

We will now utilize the generalized architecture to design a simplistic trust establishment model named the Generalized Trust Establishment Model (GTEM). GTEM contains both a postprocessing module and a toggleable preprocessing module for increased performance.

\subsection{Trustor-level Postprocessing Module Design}

Following ITE's structure but simplifying the approach and using unique equations, GTEM is a simplistic trust establishment model which uses ETME's idea of classifying trustors to help adjust a trustee's behaviours. In GTEM, trustee y classifies trustor x for task s with $t_{x}^{y}(s)$, where the trustor is either classified as overcompensated, when $t_{x}^{y}(s)=0$, or as undercompensated, when $t_{x}^{y}(s)=1$, at a time step. GTEM, like ITE, also uses predicted rw values and updates them after a transaction with the equation below.

$$
r w_{x}^{y}\left(s_{c_{i}}, r e q\right)= \begin{cases}(1-\alpha) * r w_{x}^{y}\left(s_{c_{i}}, t r a^{\prime}\right) & t_{x}^{y}(s)=0 \\ (1+\alpha) * r w_{x}^{y}\left(s_{c_{i}}, t r a^{\prime}\right) & t_{x}^{y}(s)=1\end{cases}
$$

where,

- $r w_{x}^{y}\left(s_{c_{i}}, r e q\right)$ is the predicted $\mathrm{rw}$ value between trustee $\mathrm{y}$ and trustor $\mathrm{x}$ for $s_{c_{i}}$, for interaction $r e q$, which is the interaction following the previous transaction $t r a^{\prime}$

- $\alpha$ is the variable representing the learning rate which a trustee uses with GTEM

GTEM uses these predicted rw values to derive an explicit improvement value in the same manner as ITE [3]. The explicit improvement value and the continuously updated implicit improvement value (see equation (4.2) below) are used to calculate the $\operatorname{Improvement}_{x}^{y}\left(s_{c_{i}}\right)$ value used by ITE to then determine how much UG to spend on a transaction.

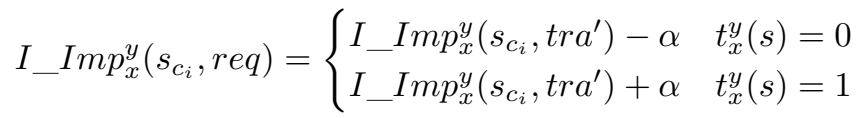

- Where $I \_I m p_{x}^{y}\left(s_{c_{i}}, r e q\right)$ is the implicit improvement to be provided by trustee y to trustor x for $s_{c_{i}}$ for the next interaction $r e q$ which follows the transaction $t r a^{\prime}$

GTEM then provides the $u g_{x}^{y}\left(s_{c_{i}}, r e q\right)$ values using equation (2.1) and the total UG value. The trustee then receives the direct feedback from the trustor via equation (2.2). These direct feedback values are used to update GTEM's $t_{x}^{y}(s)$ values. When a direct feedback value exceeds an acceptable satisfaction threshold value and the percentage of UG provided for the transaction exceeds an excessive UG threshold value, the trustor is classified as overcompensated $\left(t_{x}^{y}(s)=0\right)$. Otherwise, the trustor is classified as undercompensated $\left(t_{x}^{y}(s)=1\right)$. These $t_{x}^{y}(s)$ values help equations (4.1) and (4.2) adjust the provided UG by providing less UG to overcompensated trustors and providing more UG to undercompensated trustors. 


\subsection{Transaction-level Preprocessing Module Design}

GTEM's preprocessing module uses Hoeffding Tree online Machine Learning classifiers, defined by $\Phi_{s}$, to predict the $t_{x}^{y}(s)$ value that will be assigned to a trustor after a transaction, before completing that transaction. This prediction will be used to fine-tune the UG that will be provided to the trustor, before it is provided. The inputs used by $\Phi_{s}$ are the satisfaction values from the past two transactions between the trustor and trustee, see equation (2.2), alongside each $u g_{x}^{y}$ value to be provided to the trustor, see equation (2.1). Using these inputs, $\Phi_{s}$ outputs $p \_t_{x}^{y}(s, \operatorname{tr} a)$ which is 0 or 1 to represent the predicted trustor type.

GTEM uses $p \_t_{x}^{y}(s, t r a)$ to fine-tune the $u g_{x}^{y}$ values to be provided by the trustee. If $p \_t_{x}^{y}(s, \operatorname{tra})$ is 0, GTEM reduces a small amount that is to be provided from the $\mathrm{k}$ largest $u g_{x}^{y}$ values since the current amount is assumed to be more than what needs to be provided. Consequently, if $p_{-} t_{x}^{y}(s, \operatorname{tr} a)$ is 1, GTEM adds a small amount to the $\mathrm{k}$ lowest $u g_{x}^{y}$ values to help improve trust with the trustor. After these updates and the completion of the transaction, GTEM trains $\Phi_{s}$ to learn whether the initial inputs resulted in $p_{-} t_{x}^{y}(s, \operatorname{tr} a)$ being assigned to the trustor. This finalizes the simplistic preprocessing module which complements GTEM's postprocessing module without affecting the postprocessing process.

\section{Performance}

Next, we will define the simulation environment which compares four trust establishment models on various metrics to evaluate which models perform best.

\subsection{Simulation Definition}

To showcase GTEM's performance, GTEM is compared to itself without the preprocessing module (referred to as GTEM-Lite), ITE, and ETME over three tests. Each test is run ten times per model, where specific random seeds setup the tests identically. ETME will manage a single criteria task while the others will manage a task with ten criteria. All simulations are implemented in Python with the Mesa Multi-Agent Modelling library [10].

Like in ITE's paper, trustors contain activity levels, which dictate their chance to search for interactions at a time step, and demand levels, which specify their demands and $d_{x}\left(s_{c_{i}}\right)$ values [3]. A transaction is rejected if the proposed UG multiplied by the trustor's trust does not meet the trustor's demand level. Fully meeting a trustor's demands for each criterion results in a good transaction, otherwise it is a bad transaction. Active trustors randomly select between 1 and 30 of their most trusted trustees to interact with at time step $t$.

A trustor's trust for a trustee equals the evenly weighted combination of their direct and indirect trust of that trustee. The indirect trust is the average of the direct trust values, seen in the equation below, for the trustee from all other trustors in the environment.

$$
\text { direct_trust }_{x}^{y}= \begin{cases}1.2 *\left(\frac{\sum_{i=1}^{n} S A T_{x}^{y}(i)}{n}\right) & \text { the last five transactions were good } \\ 0.8 *\left(\frac{\sum_{i=1}^{n} S A T_{x}^{y}(i)}{n}\right) & \text { the last five transactions were bad } \\ \frac{\sum_{i=1}^{n} S A T_{x}^{y}(i)}{n} & \text { else }\end{cases}
$$

- Where $S A T_{x}^{y}(i)$ is the $\mathrm{i}^{\text {th }} \mathrm{SAT}$ value from the $\mathrm{n}$ transactions between $\mathrm{x}$ and $\mathrm{y}$

Equation (5.1) uses multipliers since logical trustors will see trustees providing consistent good or bad transactions as more or less trusted. This makes the results more comparable to when using robust trust evaluation. This is performed when the last five transactions are good or bad to help trustors quickly learn which trustees satisfy their demands and know to interact with new trustees if their demands are not being met. The $20 \%$ multiplier allows trustors to shift their trust values to remain frequently satisfied and allows trustees who provide good and bad transactions to still compete to be a trusted interaction partner. 


\subsection{Simulation Results}

Each performed test uses different trustor to trustee ratios to test diverse environments. The first test uses 12 trustors and 88 trustees to simulate a competitive environment, the second uses 36 trustors and 64 trustees to be more balanced, and the final test uses 88 trustors and 12 trustees to be less competitive but have many trustors. Trustors randomly receive an activity and demand level, where each level has a limited number of assignments, to have varying trustor behaviours in the ten runs controlled by random seeds. The average of all direct trust values, the average of all provided UG, and the rate of good transactions from all transactions are tracked in these tests. A higher trust indicates better performance when not providing excessive UG. Higher good transaction rates imply that trustees are better understanding trustor needs. The averaged results from the ten simulations run for each test, where the trustors use various demand and activity levels, are displayed below.

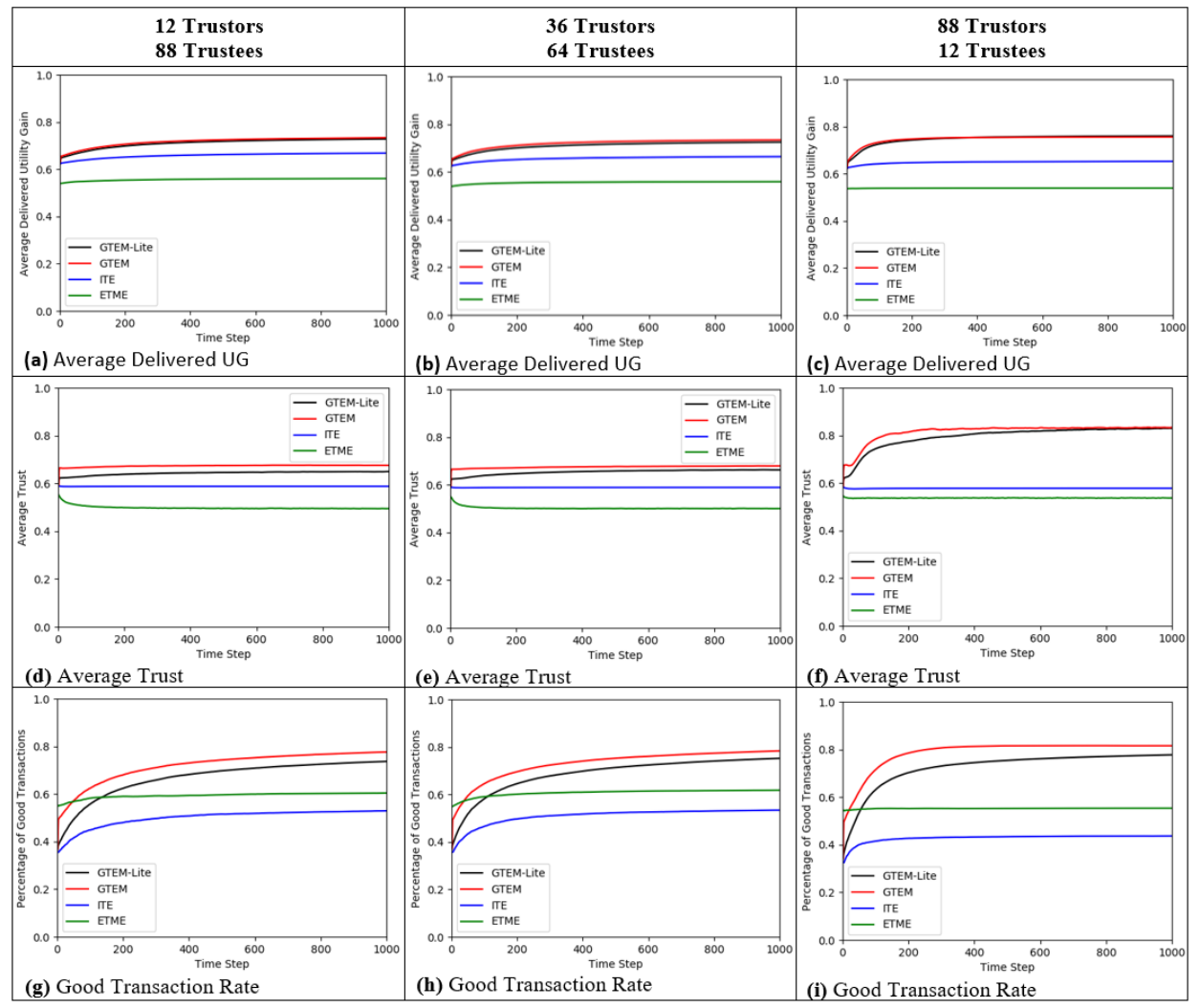

Figure 1. Comparative results from simulated tests

Figure 1 highlights that GTEM and GTEM-Lite perform better overall when compared to ITE and ETME in the tests. With less trustors and more competition, GTEM and GTEMLite have the highest trust values (plot (d)) and good transaction rates (plot (g)). This requires more provided UG (plot (a)), however GTEM and GTEM-Lite's good transaction rates are the highest. Thus, GTEM and GTEM-Lite are better at meeting trustor needs. In plot (d), ETME's trust decreases, exhibiting that its inability to adapt to specific trustor behaviours is problematic in diverse environments. Similarly, ITE's desire save UG results in a low good transaction rate when working with diverse trustors. The second test exhibits similar results when compared to the first test, see plots (b), (e), and (h). This implies that most trustor to trustee ratios with the majority as trustees will see similar results. 
In the final test, GTEM and GTEM-Lite see a large increase in average trust (plot (f)) and a minor increase in average provided UG (plot (c)). Here, GTEM and GTEM-Lite perform marginally better than ITE and ETME when comparing the average trust and good transaction rates. The average delivered UG from plot (c) resembles plots (a) and (b), but the increased good transaction rate (plot (i)) and average trust (plot (f)) exhibit that GTEM and GTEM-Lite perform best with less competition and with many trustors.

\section{Discussion and Conclusion}

Overall, the results between GTEM and GTEM-Lite end similarly in most of the tests. This is exhibited by the average trust and average delivered UG plots. Despite providing UG comparable to GTEM-Lite, GTEM sees increased trust in the first two tests (plots (d) and (e)) and until the results converge in the final test (plot (f)). This implies that despite adjusting the UG, the preprocessing module balances the UG to achieve higher trust values.

In plots (g), (h), and (i), GTEM achieves a higher good transaction rate quicker than GTEM-Lite. This means that GTEM's preprocessing is helping it more accurately meet trustor desires. Also, in plot (f) GTEM reaches the convergence point of average direct trust faster than GTEM-Lite, exhibiting faster learning speeds. This increased learning speed is important in MASs in which agents can constantly shift their behaviours since it can help a model reduce the amount of trust lost and UG spent when adjusting itself.

In conclusion, GTEM's preprocessing module improves its performance when compared to GTEM-Lite. Using the generalized architecture, a model can be more modular and can see improved performance by using a preprocessing module. By sharing and generalizing the preprocessing components, all models can see improved overall performance.

\section{References}

[1] H. Yu, Z. Shen, and C. Leung. "From Internet of Things to Internet of Agents". In: 2013 IEEE International Conference on Green Computing and Communications and IEEE Internet of Things and IEEE Cyber, Physical and Social Computing. 2013, pp. 1054-1057.

[2] S. Sen. "A Comprehensive Approach to Trust Management". In: Proceedings of the 2013 International Conference on Autonomous Agents and Multi-Agent Systems. AAMAS '13. St. Paul, MN, USA: International Foundation for Autonomous Agents and Multiagent Systems, 2013, pp. 797-800. ISBN: 9781450319935.

[3] A. Aref and T. Tran. "An integrated trust establishment model for the internet of agents". In: Knowledge and Information Systems 62 (Jan. 2020), pp. 1-27.

[4] A. Aref and T. Tran. "RLTE: A Reinforcement Learning Based Trust Establishment Model". In: 2015 IEEE Trustcom/BigDataSE/ISPA. Vol. 1. 2015, pp. 694-701.

[5] A. Aref and T. Tran. "Acting as a Trustee for Internet of Agents in the Absence of Explicit Feedback". In: MCETECH 2017. May 2017, pp. 3-23. ISBN: 978-3-319-59040-0.

[6] A. Aref and T. Tran. "FTE: A Fuzzy Logic Based Trust Establishment Model for Intelligent Agents". In: 2015 IEEE/WIC/ACM International Conference on Web Intelligence and Intelligent Agent Technology (WI-IAT). Vol. 2. 2015, pp. 133-138.

[7] C. Burnett, T. Norman, and K. Sycara. "Trust Decision-Making in Multi-Agent Systems." In: Jan. 2011, pp. 115-120.

[8] T. Tran, R. Cohen, E. Langlois, and P. Kates. "Establishing Trust in Multiagent Environments: Realizing the Comprehensive Trust Management Dream". In: TRUST@ AAMAS 1740 (2014), pp. 35-43.

[9] Y. Levine, B. Lenz, O. Dagan, O. Ram, D. Padnos, O. Sharir, S. Shalev-Shwartz, A. Shashua, and Y. Shoham. "SenseBERT: Driving Some Sense into BERT". In: CoRR abs/1908.05646 (2019). arXiv: 1908.05646. URL: http://arxiv.org/abs/1908.05646.

[10] D. Masad and J. Kazil. "Mesa: An Agent-Based Modeling Framework". In: Jan. 2015, pp. 5158 . 